\title{
Prognostic and clinical significance for the combination of systemic inflammation response index and platelet-lymphocyte ratio in patients with adenocarcinoma of the esophagogastric junction and
} upper gastric cancer

\author{
Xin Yin ${ }^{1}$, Tianyi Fang ${ }^{1}$, Xuan Lin $^{2}$, Xiliang $\mathrm{Cong}^{3}$, Yimin Wang ${ }^{1}$, Chunfeng $\mathrm{Li}^{3}$, Yan $\mathrm{Ma}^{3}$, \\ and Yingwei $\mathrm{Xue}^{1}$ \\ ${ }^{1}$ Harbin Medical University Third Hospital \\ ${ }^{2}$ Second Affiliated Hospital of Harbin Medical University \\ ${ }^{3}$ Harbin Medical University Third Clinical College
}

July 13, 2020

\begin{abstract}
Abstract Background Tumor immunity plays an important role in assessing the tumor progression. The purpose of this study was to investigate the prognostic value of combined systemic inflammation response index (SIRI) and platelet-lymphocyte ratio (PLR) for treatment of gastroesophageal junction cancer (AEG) and upper gastric cancer (UGC). Methods In this retrospective cohort study, patients from 2003 to 2014 were divided into training set $(\mathrm{n}=194)$ and validation set $(\mathrm{n}=177)$. The prognostic accuracy of each variable was compared using time-ependent ROC analysis. The scoring system was calculated by cut-off values of SIRI and PLR by ROC curve for survival in 5 years. Kaplan-Meier and Log-rank tests were used to analyze overall survival (OS). The chi-square test was used to analyze the association between clinical characteristics and the scoring system. Univariate and multivariate analyses based on the competitive risk regression model were used to analyze independent predictors of death due to AGC and UGC. The R software was used to construct the Nomogram model of risk assessment. Results Patients with SIRI-PLR=2 had worse survival time than those with 0 and $1(\mathrm{P}<0.001)$ and more suitable for postoperative adjuvant chemotherapy $(\mathrm{P}=0.003)$ and proximal gastrectomy $(\mathrm{P}=0.045)$. SIRI and PLR were independent predictors in training set $(\mathrm{P}=0.036, \mathrm{P}=0.045)$, which could be combined with age and pTNM to construct Nomogram for predicting OS. Conclusions Preoperative SIRI-PLR score was an independent predictor for patients with AEG and UGC. The Nomogram model constructed by age, SIRI, PLR and pTNM can correctly predict the prognosis of patients.
\end{abstract}

\section{Hosted file}

Text.DOCX available at https://authorea.com/users/300541/articles/469201-prognostic-andclinical-significance-for-the-combination-of-systemic-inflammation-response-indexand-platelet-lymphocyte-ratio-in-patients-with-adenocarcinoma-of-the-esophagogastricjunction-and-upper-gastric-cancer 